\title{
Retrospective analysis between complication and nipple areola complex preservation in direct-to-implant breast reconstruction
}

\author{
Jun-Ho Lee", Manki Choi, Yong Sakong ${ }^{\#}$ \\ Department of Plastic and Reconstructive Surgery, Yeungnam University Hospital, Yeungnam University College of Medicine, Daegu, Korea \\ Contributions: (I) Conception and design: JH Lee; (II) Administrative support: None; (III) Provision of study materials or patients: JH Lee; (IV) \\ Collection and assembly of data: MK Choi, Y Sakong; (V) Data analysis and interpretation: MK Choi, Y Sakong; (VI) Manuscript writing: All \\ authors; (VII) Final approval of manuscript: All authors. \\ \#These authors contributed equally to this work. \\ Correspondence to: Jun-Ho Lee, MD. Department of Plastic and Reconstructive Surgery, Yeungnam University College of Medicine, 170 \\ Hyeonchung-ro, Nam-gu, Daegu 42415, Korea. Email: junojunho@gmail.com.
}

\begin{abstract}
Background: Since the introduction of skin-sparing mastectomy (SSM), more breast surgeons have been preserving the areola and often nipple areolar complex (NAC) entirely. For better outcomes, more studies are necessary to analyze whether preserving the areola or NAC is unfavorable in breast reconstruction. The aim of this study was to assess the potential risk of areola or NAC preservation in direct-to-implant (DTI) breast reconstruction after SSM.

Methods: We retrospectively reviewed the medical records of patients who underwent immediate breast reconstruction from May 2011 to July 2017. Immediate breast reconstruction was performed with DTI procedure in all cases. In total, 213 breasts met the inclusion criteria and were divided into 3 groups: nipplesparing mastectomy (NSM), aerola-sparing mastectomy (ASM) which only nipple is excised, and skin-sparing mastectomy (SSM) which whole NAC was excised. Complications including infection, capsular contracture, skin necrosis and explantation were measured.

Results: The mean patient age was 45.3 years, with a range of 27-62 years; the mean follow-up was 3.08 years. NSM, ASM and SSM groups consisted of 121, 30 and 62 breasts, respectively. The Infection rates were statistically lower in SSM group (4.8\%) compared with NSM group (15.7\%) (P=0.033) but not statistically different from ASM group (13.3\%) ( $\mathrm{P}=0.210)$. SSM group showed significantly lower rate of overall complication compared with both NSM and ASM groups ( $\mathrm{P}=0.005,0.025$ respectively).
\end{abstract}

Conclusions: Our research suggests that preservation of the NAC increases the rate of infection and skin necrosis in DTI breast reconstruction.

Keywords! Breast reconstruction; nipple areola complex (NAC); infection

Submitted Jul 13, 2020. Accepted for publication Nov 09, 2020.

doi: 10.21037/gs-20-606

View this article at: http://dx.doi.org/10.21037/gs-20-606

\section{Introduction}

The mastectomy procedure has evolved since the era of Halstead's radical mastectomy. The modified radical mastectomy has made it possible for the development of breast conservation techniques using principles of skin preservation. Today, this advancement manifests as skin-sparing and nipplesparing mastectomies. Currently, more surgeons have been preserving the areola or nipple areola complex (NAC) due to its cosmetic advantages and comparable oncologic safety (1-3). However, postoperative infection is a possible complication in "Direct-to-implant" (DTI) breast reconstruction after skinsparing mastectomy (SSM) (4).

Like breast reconstruction using implants, bacterial infection is one of the major complications in foreign body insertion surgery. Symptoms of breast infection include 
abscess or seroma collection, erythema, pain, and wound dehiscence, and sepsis can seriously occur. Also, many plastic surgeons insert the breast implant with acellular dermal matrix (ADM) together. This ADM can result in capsular contracture, which is one of the most frequent complication in breast augmentation or reconstruction. The cause of capsular contracture is multifactorial, but chronic inflammation is known be the main cause. When capsular contracture appears, cosmetic problems such as skin rippling appear.

These infections may be caused by infection from outside the surgical site during or after surgery, but some arguments have suggested that the ductal system of the nipple areola complex in the breast may be the cause. There are normal bacterial organisms in the ductal system of the breast $(5,6)$. In particular, Staphylococcus epidermidis and Bacillus subtilis appeared mostly in the nipple duct system. Although these bacteria are not pathogenic, in foreign body insertion surgery such as implantation, they can form a biofilm on the breast implant surface and cause infection. It has also been reported that several bacterial species appear throughout breast tissue as well as nipple areola complex, with or without breastfeeding (7).

Authors reported somewhat high rates of complications due to infection in direct-to-implant breast reconstruction after nipple-sparing mastectomy (NSM), when compared with SSM with NAC excision. We hypothesized that this could have resulted from microbial spread through the mammary duct, a possible source of infection, while inserting the breast implant. Moreover, concerns regarding the possible impact of poor vascularity of the NAC on nipple and skin necrosis were also investigated in this study.

The aim of this study was to assess the impact of the areola or NAC preservation in DTI immediate breast reconstruction. We assessed postoperative complications of breast reconstruction following NSM, areola-sparing mastectomy (excision of nipple) and SSM with NAC excision. In the context of this study, NSM indicates SSM with NAC preservation, while areola-sparing mastectomy means SSM with areola preservation without the nipple. We present the following article in accordance with the STROBE reporting checklist (available at http://dx.doi. org/10.21037/gs-20-606).

\section{Methods}

A retrospective cohort study of DTI breast reconstruction after skin-sparing mastectomy was started in 2011. The medical records of patients who underwent immediate breast reconstruction following skin-sparing mastectomy from May 2011 to July 2017 were collected. The study was conducted in accordance with the Declaration of Helsinki (as revised in 2013). The study was approved by institutional review board of Yeungnam University Hospital (NO. 202006-072) and informed consent were taken from all the patients of clinical photographs at the figure.

The inclusion criteria included one-stage DTI breast reconstruction with breast implant and Acellular dermal matrix (ADM) after SSM. We excluded the following patients: those with two-stage breast reconstruction; those with autologous reconstruction after SSM; those with preoperative radiation; less follow up than 3 months; and those who expect to be exposed to radiation postoperatively.

In total, 213 breasts fulfilled the inclusion criteria. The study group was divided into three groups: Nipple sparing mastectomy (NSM) group, areola-sparing mastectomy (ASM) group, and skin-sparing mastectomy (SSM) group. NSM group was designed as reconstructed breasts after nipple-sparing mastectomy (preservation of NAC). ASM group was designed as reconstructed breasts after areolasparing mastectomy (preservation of areola only, excising the nipple). SSM group was designed as reconstructed breasts after skin-sparing mastectomy with NAC excision (Figure 1).

Indications for NAC preservation was based on the clinical decision in accordance with the pathologic and radiographic involvement of NAC (8). While performing mastectomy, the retro-areola margin was taken from the nipple and saved as a frozen section (9). A positive frozen section was treated with nipple removal or removal of the whole NAC. Determination for areola preservation was made intraoperatively based on the experience of the breast surgeon.

All patients had mastectomy from two breast surgeons. Two breast surgeon performed mastectomy with least variation of surgical technique. After undergoing mastectomy, immediate breast reconstruction was performed with DTI reconstruction in all cases by single surgeon of plastic surgery department. Acellular dermal matrix was used in all cases. During this study, all patients underwent DTI immediate breast reconstruction with one of the three types of acellular dermal matrix (ADM); CryoDerm (CG BIO Corp., Seongnam, Korea), AlloDerm (LifeCell Corp., Branchburg, NJ, USA), and MegaDerm (L\&C Bio, Seoul, Korea) and one of the two types of breast implants (Anatomical silicone implant or Textured silicone implants, Allergan Corp., Irvine, CA, USA). 


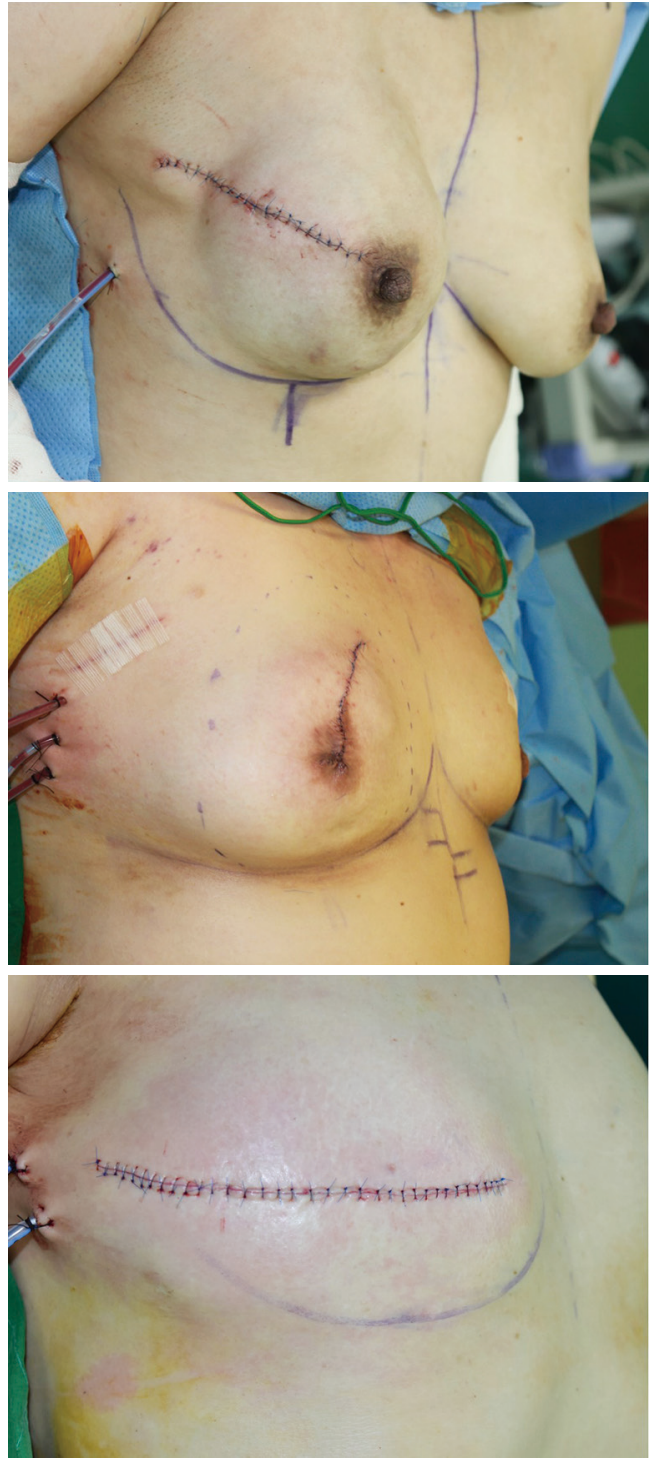

Figure 1 Classification of the cohorts. (Above) Postoperative photo of NSM; nipple-sparing mastectomy (Middle) postoperative photo of ASM; areolar-sparing mastectomy, and (Below) postoperative photo of SSM; skin-sparing mastectomy.

Patient characteristics, including patient age, body mass index, comorbid conditions, volume of resected tissue, distance from nipple to sternal notch, operation time, duration for drainage, follow-up, size of implant, adjuvant chemotherapy, and ADM were examined (Table 1). The outcome measures included complications, which included infection, capsular contracture, skin/nipple necrosis, and explantation within 3 months after DTI operation (Table 2). Depending on the stage of breast cancer, postoperative treatment (chemotherapy, radiation therapy, hormone therapy) is different for each patient, and the time taken to start these treatments is different. Therefore, to minimize the effect of postoperative treatment on complication, infection and skin necrosis included only infections that occurred within 3 weeks after surgery. Skin necrosis include full-thickness necrosis and partial-thickness necrosis. Reconstructive surgeon evaluated the flap state through skin flap color, capillary refill, temperature, turgor, and dermal bleeding. Baker grades of III and IV were regarded as capsular contractures.

\section{Statistical analysis}

Statistical analysis was performed using by SPSS Version 19.0. Patient-related characteristics were compared among the two Cohorts. Descriptive statistics were used to compare the postoperative complications, including infection, capsular contracture, skin necrosis, explantation, and total nipple necrosis, across three groups. The Pearson chi-square test was performed to analyze categorical variables and to check for correlations between NAC preservation and outcomes. A value of $\mathrm{P}<0.05$ indicated statistical significance.

\section{Results}

From 209 patients, there were four cases of bilateral reconstruction; thus, 213 mastectomy procedures were performed in total. The mean patient age was 45.3 years, with a range of 27-62 years; the mean follow-up was 3.08 years. Table 1 presents patient characteristics. We compared the three groups, separately, but no statistically significant differences were observed. Of the 213 patients, 121 patients were in the NSM group, 30 patients in the ASM group, and 62 patients in the SSM group, respectively. Table 2 shows the complications among patients across the three groups, including infection, capsular contracture, skin necrosis, and explantation. NSM group exhibited a significantly higher rate of infection and overall complication in comparison to SSM group, whereas ASM group exhibited a significantly higher rate of overall complication in comparison to SSM group. There were no statistically significant differences in overall complications between NSM and ASM group (Tables 2,3). For the main comparison between patients NSM group and SSM group, the infection rates were significantly lower in the SSM group (4.8\%) than in NSM 
Table 1 Demographic of three cohorts

\begin{tabular}{|c|c|c|c|c|}
\hline & NSM & ASM & SSM & $P$ values \\
\hline \multicolumn{5}{|l|}{ Age, years } \\
\hline Mean & 45.5 & 45.9 & 44.7 & 0.308 \\
\hline Range & $28-62$ & $28-60$ & $27-62$ & \\
\hline Mean & 23.7 & 22.8 & 23.1 & 0.217 \\
\hline Range & $17.7-30.1$ & $18.5-32.3$ & $17.6-31.8$ & \\
\hline \multicolumn{5}{|l|}{ Comorbid conditions } \\
\hline Smoking & $3(2.5)$ & $1(3.3)$ & $2(3.2)$ & 0.853 \\
\hline \multicolumn{5}{|l|}{ Volume of resected tissue (cc) } \\
\hline Mean & 273.3 & 277.5 & 259.2 & 0.955 \\
\hline Range & $50-1,030$ & $50-640$ & $75-580$ & \\
\hline \multicolumn{5}{|l|}{ Operation time (min) } \\
\hline Mean & 168.4 & 164.6 & 186.5 & 0.821 \\
\hline Range & $75-217$ & $62-239$ & $65-226$ & \\
\hline \multicolumn{5}{|l|}{ Duration for drainage (day) } \\
\hline Mean & 15.8 & 16.7 & 16.2 & 0.526 \\
\hline Adjuvant chemotherapy & $65(53.7)$ & $14(46.7)$ & $32(51.6)$ & 0.61 \\
\hline Neoadjuvant chemotherapy & $5(4.13)$ & $0(0)$ & $4(6.45)$ & 0.340 \\
\hline Post-mastectomy radiotherapy & $4(3.31)$ & $1(3.33)$ & $10(16.13)$ & 0.006 \\
\hline \multicolumn{5}{|l|}{ Acellular dermal matrix } \\
\hline Alloderm & $16(13.3)$ & $5(16.7)$ & $10(16.1)$ & 0.764 \\
\hline Cryoderm & $42(34.7)$ & $13(43.3)$ & $34(54.8)$ & 0.017 \\
\hline Megaderm & $63(52.0)$ & $12(40.0)$ & $18(29.1)$ & 0.040 \\
\hline
\end{tabular}

NSM, nipple-sparing mastectomy; ASM, areolar-sparing mastectomy; SSM, skin-sparing mastectomy. Values are presented as number (\%)




Table 2 Rates of complications in the three cohorts

\begin{tabular}{lcccc}
\hline & NSM (\%) & ASM (\%) & SSM (\%) & P values \\
\hline Infection & $19(15.7)$ & $4(13.3)$ & $3(4.8)$ & 0.089 \\
Capsular contracture & $4(3.3)$ & $1(3.3)$ & $1(1.6)$ & 0.732 \\
Skin necrosis & $13(10.7)$ & $3(10.0)$ & $1(1.6)$ & 0.156 \\
Cases of explantation & $9(7.4)$ & $1(3.3)$ & $1(1.6)$ & 0.223 \\
Overall complication & $36(29.8)$ & $8(26.6)$ & $5(8.0)$ & 0.009 \\
\hline
\end{tabular}

NSM, nipple-sparing mastectomy; ASM, areolar-sparing mastectomy; SSM, skin-sparing mastectomy.

Table 3 Pearson Chi square analysis of complications among the three cohorts

\begin{tabular}{lccc}
\hline & \multicolumn{3}{c}{ P values } \\
\cline { 2 - 4 } & NSM:ASM & ASM:SSM & NSM:SSM \\
\hline Infection & 1.000 & 0.210 & 0.033 \\
Capsular contracture & 1.000 & 0.548 & 0.664 \\
Skin necrosis & 1.000 & 0.100 & 0.037 \\
Cases of explantation & 0.688 & 0.548 & 0.168 \\
Overall complications & 0.825 & 0.025 & 0.001 \\
\hline
\end{tabular}

NSM, nipple-sparing mastectomy; ASM, areolar-sparing

mastectomy; SSM, skin-sparing mastectomy.

group $(15.7 \%)(\mathrm{P}=0.033)$, but not significantly different from ASM group (13.3\%) $(\mathrm{P}=0.21)$. SSM group showed a significantly low rate of skin necrosis $(1.6 \%)$ compared to the NSM group (6.6\%) $(\mathrm{P}=0.037)$. With respect to capsular contracture, there were no statistical differences across the three groups. Regarding major complications, explantation of implant occurred in nine patients in NSM group and one patient each in ASM and SSM group. However, these rates were not statistically different $(\mathrm{P}>0.05)$.

\section{Discussion}

We experienced a relatively high rate of skin necrosis in cases undergoing DTI breast reconstruction after NSM when compared with after SSM. Thus, we hypothesized that this could be caused by vascular vulnerability in NAC. The intercostal artery was regarded as the main and constant contributor of blood supply to the NAC via its perforators and anterior intercostal branches (10). Depending on its variable anatomical patterns of blood supply, in some cases, nipple vascularity may potentially be compromised and result in nipple necrosis (10-13). In this perspective, the higher rates of skin necrosis in NSM group, compared with SSM group, could be explained by the possible destruction of the nipple vasculature while there is excessive tension or dissection at the base of NAC (14). After mastectomy, the dermal flap becomes thinner, and flap vascularity is regarded as a very important factor for the proper healing of flaps (15). Damage, formation of a hypovascular subdermal plexus, or local perforation of the NAC and periareolar region may result in delayed healing and skin necrosis (16).

Additionally, the lactiferous duct could contribute to the increasing tendency of postoperative infection in NSM followed by DTI breast reconstruction. Previous studies have shown that bacterial strains reside in the peri-nipple area (17-19) and the lactiferous duct may play a role in the transfer of bacteria from the skin to the breast parenchyma while handling the nipple and areola. Therefore, the underlying cause of infection in immediate breast reconstruction with DTI following skin-sparing mastectomy may arise in the process of NAC preservation. The complication rate of infection was significantly lower in SSM group. Such results are noteworthy considering the risk of transductal transfer of bacteria. We do not routinely perform wound culture. We assessed the pattern of discharge, the pattern of skin color around wound, the healing state of wound, and the presence of a fever. Explantation was observed in all cases in the infection group, which highlights the importance of exercising caution when operating on the nipples.

Several studies have discussed how to block exposure of NAC through a nipple areola shield $(14,20)$. N. Collis applied adhesive filme on NAC in preventing perioperative expression of bacteria, contaminating the operative field. $33 \%$ of breasts yielded bacterial growth form swabs under the film postoperatively. Roger also reported the exposed nipple during breast augmentation is potential source of implant contamination. He studied 63 nipples in breast augmentation and nipple shields yielded $34.9 \%$ of positive for bacterial contamination. On the basis of these findings, we suggest that caution should be exercised when managing DTI breast reconstruction combined with nipplesparing mastectomy. Such procedures include: the aseptic preoperative drape technique, preoperative coverage of the NAC and umbilicus with surgical film, and handling of breast and NAC with minimal tension. Moreover, an omega-shape periareolar skin incision and anchoring suture are effective ways to decrease suture site tension and prevent skin necrosis (21). 
Additionally, irrigation using a solution of antibiotics in combination with postoperative hyperbaric therapy is useful for avoiding intraoperative infection and skin flap ischemia. Povidone-iodine irrigation shown effective prevention of surgical site in other general surgery (22). In Adams' invitro study, the combination breast irrigation of bacitracin, cefazolin, and gentamicin reported effective control of bacteria (23). In subsequent clinical studies, the capsular contraction rate was reported to be reduced to four to five time (24). Pfeiffer et al. reported decrease of infection in cosmetic breast surgery by using antibiotic mixed saline irrigation in breast pocket (25). There are slight differences depending on the irrigation method after implantation, but antibiotic and povidone mixed irrigation has little effect on the implant and reduces inflammation and infection (26).

Postoperative hyperbaric oxygen treatment might be used effectively by optimizing fibroblast proliferation and collagen synthesis, for that, it has been used in diabetic foot, crush injury, radiation-induced injury and burn wound (27-29). In this study, all patient who had postoperative skin necrosis went through hyperbaric oxygen treatment at 2 ATA for 60 minutes. Surgical debridement was done if necessary. Although this study does not show that hyperbaric oxygen treatment critically prevent skin necrosis, previous studies suggest that it can be used to prevent and treat skin necrosis. Also, unequal sample size of each cohort and retrospective character limits statistical refinement. Breast skin flap thickness after is major factor of skin necrosis. It would be better to evaluate the complication rate with breast flap thickness. Some study obtained bacterial culture from nipple areola complex. In a further study, it is necessary to confirm which bacteria are detected by performing culture inside the breast when reoperation is performed in a patient with breast infection. Further, we will confirm that these bacteria are the same as those detected in the nipple areola complex.

This study did not have any significant difference of complication when the three ADM types were compared. There was no significant difference in each group by examining the underlying disease and postoperative treatment of patients in the demographic of the three groups. Therefore, in future studies, it is necessary to compare complications by matching the patient's underlying disease, $\mathrm{ADM}$, postoperative treatment method, and timing as a prospective method.

\section{Conclusions}

Nipple sparing mastectomy tended to show higher rates of infection and skin necrosis than skin sparing mastectomy. On the other hand, there was no difference in the incidence of complication when excision of nipple only and compared with areola sparing group. Nipple areola complex is an important cosmetic component of the breast, and reconstruction is possible after resection. Therefore, it should be performed after sufficient consultation with the patient for prophylactic excision.

\section{Acknowledgments}

Funding: This study was funded by the 2018 Yeungnam University research grants.

\section{Footnote}

Reporting Checklist: The authors have completed the STROBE reporting checklist. Available at http://dx.doi. org/10.21037/gs-20-606

Data Sharing Statement: Available at http://dx.doi. org/10.21037/gs-20-606

Peer Review File: Available at http://dx.doi.org/10.21037/gs20-606

Conflicts of Interest: All authors have completed the ICMJE uniform disclosure form (available at http://dx.doi. org/10.21037/gs-20-606). The authors have no conflicts of interest to declare.

Ethical Statement: The authors are accountable for all aspects of the work in ensuring that questions related to the accuracy or integrity of any part of the work are appropriately investigated and resolved. The study was conducted in accordance with the Declaration of Helsinki (as revised in 2013). The study was approved by institutional review board of Yeungnam University Hospital (NO. 202006-072) and informed consent were taken from all the patients of clinical photographs at the figure.

Open Access Statement: This is an Open Access article distributed in accordance with the Creative Commons Attribution-NonCommercial-NoDerivs 4.0 International 
License (CC BY-NC-ND 4.0), which permits the noncommercial replication and distribution of the article with the strict proviso that no changes or edits are made and the original work is properly cited (including links to both the formal publication through the relevant DOI and the license). See: https://creativecommons.org/licenses/by-nc-nd/4.0/.

\section{References}

1. de Alcantara Filho P, Capko D, Barry JM, et al. Nipplesparing mastectomy for breast cancer and risk-reducing surgery: the Memorial Sloan-Kettering Cancer Center experience. Ann Surg Oncol 2011;18:3117-22.

2. Spear SL, Willey SC, Feldman ED, et al. Nipple-sparing mastectomy for prophylactic and therapeutic indications. Plast Reconstr Surg 2011;128:1005-14.

3. Kim HR, Lim JS, Kim SM, et al. One-stage nipple and breast reconstruction following areola-sparing mastectomy. Arch Plast Surg 2013;40:553-8.

4. Hunsicker LM, Ashikari AY, Berry C, et al. Short-Term Complications Associated With Acellular Dermal MatrixAssisted Direct-to-Implant Breast Reconstruction. Ann Plast Surg 2017;78:35-40.

5. Thornton JW, Argenta LC, McClatchey KD, et al. Studies on the endogenous flora of the human breast. Ann Plast Surg 1988;20:39-42.

6. Mladick RA. Significance of Staphylococcus epidermidis causing subclinical infection. Plast Reconstr Surg 2005;115:1426-7.

7. Urbaniak C, Cummins J, Brackstone M, et al. Microbiota of human breast tissue. Appl Environ Microbiol 2014;80:3007-14.

8. Colwell AS, Tessler O, Lin AM, et al. Breast reconstruction following nipple-sparing mastectomy: predictors of complications, reconstruction outcomes, and 5-year trends. Plast Reconstr Surg 2014;133:496-506.

9. Colwell AS, Gadd M, Smith BL, et al. An inferolateral approach to nipple-sparing mastectomy: optimizing mastectomy and reconstruction. Ann Plast Surg 2010;65:140-3.

10. van Deventer PV. The blood supply to the nipple-areola complex of the human mammary gland. Aesthetic Plast Surg 2004;28:393-8.

11. Mandrekas AD, Zambacos GJ, Anastasopoulos A, et al. Reduction mammaplasty with the inferior pedicle technique: early and late complications in 371 patients. $\mathrm{Br}$ J Plast Surg 1996;49:442-6.

12. Cárdenas-Camarena L. Reduction mammoplasty with superolateral dermoglandular pedicle: details of 15 years of experience. Ann Plast Surg 2009;63:255-61.

13. Blondeel PN, Hamdi M, Van de Sijpe KA, et al. The latero-central glandular pedicle technique for breast reduction. Br J Plast Surg 2003;56:348-59.

14. Yukun L, Ke G, Jiaming S. Application of Nipple Retractor for Correction of Nipple Inversion: A 10-Year Experience. Aesthetic Plast Surg 2016;40:707-15.

15. Vu MM, Kim JY. Current opinions on indications and algorithms for acellular dermal matrix use in primary prosthetic breast reconstruction. Gland Surg 2015;4:195-203.

16. Cho JW, Yoon ES, You HJ, et al. Nipple-Areola Complex Necrosis after Nipple-Sparing Mastectomy with Immediate Autologous Breast Reconstruction. Arch Plast Surg 2015;42:601-7.

17. Ammari FF, Yaghan RJ, Omari AK. Periductal mastitis. Clinical characteristics and outcome. Saudi Med J 2002;23:819-22.

18. Dixon JM, Thompson AM. Effective surgical treatment for mammary duct fistula. Br J Surg 1991;78:1185-6.

19. Hughes LE. Management of recurrent infection following surgery for periductal mastitis. Br J Clin Pract Suppl 1989;68:81-5; discussion 87-9.

20. Wixtrom RN, Stutman RL, Burke RM, et al. Risk of breast implant bacterial contamination from endogenous breast flora, prevention with nipple shields, and implications for biofilm formation. Aesthet Surg J 2012;32:956-63.

21. Lee JH, Hur SW, Kim YH, et al. Simple anchoring suture to minimise asymmetric nipple-areola complex (NAC) in immediate unilateral breast reconstruction with implant. J Plast Reconstr Aesthet Surg 2015;68:273-5.

22. Chundamala J, Wright JG. The efficacy and risks of using povidone-iodine irrigation to prevent surgical site infection: an evidence-based review. Can J Surg 2007;50:473-81.

23. Adams WP, Jr., Conner WC, Barton FE Jr, et al. Optimizing breast-pocket irrigation: the post-betadine era. Plast Reconstr Surg 2001;107:1596-601.

24. Adams WP, Jr., Rios JL, Smith SJ. Enhancing patient outcomes in aesthetic and reconstructive breast surgery using triple antibiotic breast irrigation: six-year prospective clinical study. Plast Reconstr Surg 2006;118:46s-52s.

25. Pfeiffer P, Jorgensen S, Kristiansen TB, et al. Protective effect of topical antibiotics in breast augmentation. Plast Reconstr Surg 2009;124:629-34.

26. Adams WP, Jr. Capsular contracture: what is it? What causes it? How can it be prevented and managed? Clin 
Plast Surg 2009;36:119-26, vii.

27. Jain KK. HBO Therapy in Wound Healing, Plastic Surgery, and Dermatology. In: Textbook of Hyperbaric Medicine. Springer, Cham 2017:183-205.

28. Tumerdem-Ulug B, Kuran I, Ozden BC, et al. Does

Cite this article as: Lee JH, Choi M, Sakong Y. Retrospective analysis between complication and nipple areola complex preservation in direct-to-implant breast reconstruction. Gland Surg 2021;10(1):290-297. doi: 10.21037/gs-20-606 hyperbaric oxygen administration before or after irradiation decrease side effects of irradiation on implant sites? Ann Plast Surg 2011;67:62-7.

29. Doctor N, Pandya S, Supe A. Hyperbaric oxygen therapy in diabetic foot. J Postgrad Med 1992;38:112. 